Yira Beatriz Alvarado Trespalacios

Magíster en Dirección Estratégica por la Universidad del Atlántico

Docente de cátedra de la Universidad del Atlántico

Colombia

[yiraalvarado@mai.uniatlantico.edu.co]

Alma Lorena Rodero Acosta Magíster en Cooperación Internacional para el Desarrollo Universidad San Buenaventura, convenio con la Universidad de Pavía (Italia) Docente y coordinadora misional de Extensión y Proyección Social de la Universidad del Atlántico Colombia [almarodero@mail.uniatlantico.edu.co]

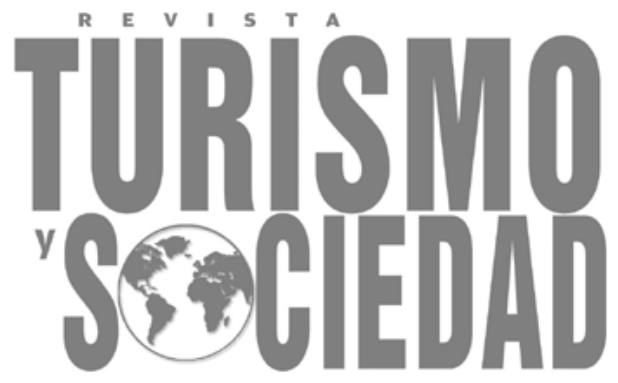

\section{MOMPOX, BOLÍVAR SUSTENTABLE Y SOSTENIBLE EN EL TIEMPO1}

\author{
MOMPOX, BOLIVAR \\ SUSTAINABLE AND \\ SUSTAINABLE OVER TIME
}

1 Para citar el artículo: Alvarado, Y. y Rodero, A. (2021). Mompox, Bolívar sustentable y sostenible en el tiempo. Turismo y Sociedad, XXVIII, pp. 39-56. DoI: https://doi org/10.18601/01207555.n28.02

Fecha de recepción: 12 de agosto de 2019

Fecha de modificación: 24 de octubre de 2019

Fecha de aceptación: 9 de diciembre de 2019

\section{Resumen}

El propósito de este trabajo consiste en elaborar un plan estratégico en turismo para el Distrito Especial, Turístico, Histórico y Cultural de Santa Cruz de Mompox, ubicado en el departamento de Bolívar (Colombia), teniendo en cuenta que la planificación estratégica es un proceso dinámico flexible que permite las modificaciones en los métodos a fin de responder a las cambiantes circunstancias que suceden en esta actividad. Hoy en día el turismo es una de las principales fuentes económicas a nivel mundial, generador de empleo e impulsor del desarrollo, por eso juega un papel fundamental como actividad capaz de promover unión entre los aspectos social y económico a nivel global. Mompox se ha convertido desde años atrás en uno de los destinos de rápido crecimiento, poderoso vínculo social e intercultural que inmiscuye a miles de personas. Metodológicamente, esta investigación se enfoca en el método cuantitativo, de tipo descriptivo, con un diseño de investigación de carácter no experimental, transaccional o transversal.

Palabras claves: Plan estratégico, turismo cultural y ecológico, sostenibilidad, Mompox.

\section{Abstract}

The purpose of this paper was to develop a strategic plan for the special touristic, historic and cultural district of Santa Cruz de Mompox, located in the department of Bolívar (Colombia). This, while bearing in mind that strategic planning is a flexible dynamic process that allows modifications in the methods in order to respond to the changing circumstances that occur within this activity. Nowadays, tourism is one of the main economic sources worldwide, generating employment, boosting development and playing a fundamental role as an activity 
capable of promoting social, economic and global union. Mompox, over the last several years, has evolved into one of the fastest growing destinations with a, powerful social and intercultural link that touches thousands of people. Methodologically, this research focuses on a quantitative method, of a descriptive nature, with a non-experimental, transactional research design.

Keywords: Strategic plan, cultural and ecological tourism, sustainability, Mompox.

\section{Introducción}

En una economía cada vez más global y competitiva como la actual, el sector turismo requiere contar con organizaciones caracterizadas por su solidez, flexibilidad e innovación, en las que se apliquen con eficiencia procesos científicos de administración y gestión que las dirijan a una toma de decisiones eficiente y a ser más competitivas en un mercado cada vez más exigente en lo que respecta a las expectativas y necesidades de sus clientes, es decir, de los turistas (De Ávila, Pérez y Vega, 2016). Por esta razón, la mayor responsabilidad de los gerentes de las empresas del sector turismo consiste en "buscar alternativas encaminadas [a] lograr rentabilidad y alcanzar una posición estratégica de liderazgo competitivo" (De Ávila, Pérez y Vega, 2016).

Ante esta realidad del turismo como una importante actividad económica, muchas regiones de Colombia reconocen los potenciales destinos turísticos que pueden fortalecer la economía de sus departamentos e intentan buscar estrategias que permitan explotar al máximo esta industria.

En tal sentido, el presente estudio propone desarrollar un diagnóstico sobre el turismo en el Distrito Especial, Turístico, Histórico y Cultural de Santa Cruz de Mompox, ubicado en el departamento de Bolívar (Colombia), el cual fue declarado patrimonio histórico y cultural de la humanidad por la UNESCO dadas sus características culturales. La intención es promover un turismo cultural y ecológico encaminado a un desarrollo sostenible en pro de generar beneficio a la comunidad mediante el impulso de emprendimientos culturales y sociales, eso sí, preservando las tradiciones con miras a beneficiar a la comunidad y ofreciendo experiencias memorables a los turistas que llegan al municipio atraídos por los imaginarios históricos con que este lugar cuenta desde la época de la Colonia.

El objetivo consiste en impulsar la creación de planes estratégicos de turismo y cuidado del medioambiente en Mompox. Para ello hay que tener en cuenta, en primer lugar, que la planificación estratégica es un "proceso dinámico lo suficientemente flexible para permitir y hasta forzar modificaciones en los métodos [empleados en la elaboración de un plan estratégico], a fin de responder a las cambiantes circunstancias" (Amador, 2002) que suceden, en este caso, en la actividad turística, la cual ofrece a la comunidad donde se lleva a cabo una oportunidad clara para su crecimiento económico, social y cultural.

En relación con el turismo en Santa Cruz de Mompox, allí se desarrollan actividades que afectan de manera directa a las zonas donde se ubican atractivos históricos y culturales, pues tales zonas se encuentran en constante crecimiento. Puede afirmarse que Santa Cruz de Mompox cuenta con amplia experiencia en el sector turístico y que allí se promueven acciones y actividades encaminadas al desarrollo turístico, cultural y ecológico caracterizadas por la presencia y relevancia de la cultura viva de su comunidad, por la identidad del territorio y por la importancia de la preservación y la sostenibilidad, con miras a lograr un equilibrio entre lo que buscan los turistas y lo que ofrece la comunidad. Ahora bien, 
hasta la fecha, el Distrito no cuenta con un estudio que, desde la investigación científica, aporte una propuesta que permita plantear a la comunidad un crecimiento sostenible en el tiempo, considerando que allí se ofrecen atractivos turísticos transcendentales, como son la historia y la cultura.

En virtud de lo mencionado, la presente investigación plantea la perspectiva según la cual tanto el turismo cultural como el ecológico pueden ser alternativas para el desarrollo $\mathrm{y}$ el beneficio económico de Santa Cruz de Mompox, de manera que la población pueda involucrarse en la creación de sus propias fuentes de ingreso, que impulse los emprendimientos culturales y ecológicos, a la vez que preserve las tradiciones históricas, todo ello en pro de generar beneficio a la comunidad.

Otros de los objetivos de esta investigación son:

- Identificar las etapas del plan estratégico de turismo para la región en cuestión.

- Validar las etapas del plan estratégico.

- Diagnosticar las actividades turísticas del Distrito Especial, Turístico, Histórico y Cultural de Santa Cruz de Mompox.

- Identificar los elementos estratégicos de distribuidoras de servicios y productos turísticos en Mompox.

- Describir los emprendimientos turísticos y ecológicos de Santa Cruz de Mompox.

Se busca que la población pueda involucrarse en la creación de sus propias fuentes de trabajo; además, Santa Cruz de Mompox cuenta con recursos naturales, culturales y ecológicos que son atractivos turísticos. En el aspecto cultural, en particular, se pretende preservar y difundir las costumbres que caracterizan a los momposinos y contribuir a la mejora en su calidad de vida mediante un desarrollo turístico viable y sostenible tanto económicamente como en el cuidado de su medioambiente.

\section{Turismo, planeación y emprendimiento estratégico: abordaje de la problemática en Santa Cruz de Mompox}

"El turismo como fenómeno social y como actividad económica es, desde hace unos años, realidad asumida" (Altamira y Muñoz, 2007, p. 679). Por esta razón, diferentes organizaciones mundiales, instituciones de Gobierno y personalidades han estudiado este fenómeno y han tratado de elaborar definiciones de este concepto desde su nacimiento, a mediados del siglo XIX, pasando por su auge y reconocimiento como actividad o negocio rentable en la década de los 50 del siglo $\mathrm{XX}, \mathrm{y}$ como actividad socioeconómica y ambientalmente reconocida desde la primera década del siglo XXI.

Para la Organización Mundial de Turismo (OMT, 2014), el turismo es la "clave para el desarrollo, la prosperidad y el bienestar social" (p. 2), es así como:

Un número creciente de destinos de todo el mundo se han abierto y han invertido en este sector, haciendo del mismo (sic) un factor clave de progreso socioeconómico mediante la obtención de ingresos por exportaciones, la creación de puestos de trabajo y de empresas y la ejecución de infraestructuras. Durante las seis últimas décadas, el turismo ha experimentado una continua expansión y diversificación, convirtiéndose (sic) en uno de los sectores económicos de mayor envergadura y crecimiento del mundo. A los destinos favoritos tradicionales de Europa y América del Norte se han sumado muchos otros. A pesar de ocasionales conmociones [y coyunturas], 
las llegadas de turistas internacionales han registrado un crecimiento prácticamente ininterrumpido: desde los 25 millones en 1950 hasta los 278 millones en 1980, los 528 millones en 1995 y los 1.087 millones en 2013. Según las previsiones a largo plazo de la OMT, incluidas en Tourism towards 2030 (Turismo hacia 2030), las llegadas de turistas internacionales a escala mundial crecerán un 3,3\% anualmente entre 2010 y 2030 hasta alcanzar los 1.800 millones en 2030. (OMT, 2014, p. 2).

Hoy en día, el turismo constituye una de las estrategias fundamentales de desarrollo de los países y es un significativo impulsor económico en el mundo, no en vano la Organización Mundial del Turismo (OMT, 2004) destaca que la industria turística y de ocio será considerada como el primer sector de la economía a nivel mundial en este siglo.

"En la actualidad cualquier destino turístico debe de especializarse en aprovechar todas las características y [los] atributos con los que cuenta para definir una oferta diferente y competitiva" (Femenía, 2011). En el caso del Caribe colombiano, este se ha consolidado como la región turística por excelencia debido a su ubicación geoestratégica, al atractivo de sus recursos y a su trayectoria.

En virtud de lo anterior, el Distrito Especial, Turístico, Histórico y Cultural de Santa Cruz de Mompox requiere un ordenamiento turístico desde la perspectiva de la planificación, la cual se entiende como un proceso en el que se definen las metas y los medios necesarios para alcanzarlas. De acuerdo con Hall y Page (2002), "la planificación deberá proveer la información necesaria para la toma de decisiones acertadas, de manera democrática y comunicada".

Se presentan a continuación las miradas de algunos tratadistas con respecto al panorama del turismo, la planificación y el emprendimiento estratégico en este sector de la economía.

En palabras de Fernández y Aharon (1995), citados en Salcedo A. (2013),

para el turismo es muy importante la planificación, cualquier actividad económica que tenga impactos significativos en la comunidad requiere de esta herramienta (sic), donde se puede anticipar en el futuro a pesar de los intereses individuales que marcan un factor crítico en la sociedad actual. (Fernández y Aharon, 1995).

Para planificar en el turismo (sic) implica generar una conciencia colectiva de la importancia de esta actividad para una región y desatar el compromiso de todos los actores involucrados en su gestión.

Así se construye participativamente un desarrollo equitativo, incluyente, innovador y sostenible desde el punto de vista ambiental.

De acuerdo con Aguilera, Bernal y Quintero (2006, p. 4), el turismo actualmente es una actividad clave en las regiones rezagadas, pues conlleva beneficios por su capacidad de potencializar el desarrollo, la modernización y la economía de las regiones. Aguilera et al. (2006) también manifiestan que el turismo plantea una serie de incentivos con respecto a la generación de divisas y la creación de oportunidades de empleo, por lo cual es considerada una industria intensiva en lo referente a la generación de trabajo.

"Sin embargo, estos beneficios pueden verse cuestionados si el turismo no se desarrolla de una manera planificada y con participación de la población local", lo que ocasiona una serie de impactos negativos, como "el deterioro del medio ambiente, debido al manejo de recursos y la construcción de infraestructura" (Aguilera et al., 2006, p. 5.), y la exclusión de los sectores tradicionales. 
En relación con lo planteado, es necesario contar con una política turística que contemple tanto los pros como los contras del desarrollo de la actividad turística en las regiones.

De acuerdo con el Ministerio de Comercio, Industria y Turismo, la política turística de Colombia tiene como objetivo optimizar la competitividad de los destinos y los productos, e incrementar la participación de la comunidad en la prestación de los servicios turísticos. Se busca que la población perciba aumentos en sus ingresos y contribuya a la generación de riqueza, a su vez que se logra una distribución equitativa y un aumento en la calidad de vida de residentes. (Aguilera et al., 2006, p. 10).

A nivel internacional, la Comisión Europea ha recomendado a las naciones adoptar medidas que animen a los ciudadanos a ser generadores de empleo, a ser emprendedores, además de cualificarlos para que sus proyectos alcancen el éxito.

Esto supone reformas en los sistemas de educación $[\ldots]$, cambios culturales y medidas para eliminar obstáculos en la creación de nuevas empresas. El fomento del espíritu emprendedor también supone el establecimiento de un entorno favorable para la creación de empresas, [...] la simplificación administrativa, una mejora del entorno normativo y financiero y el acceso a programas comunitarios como los de I+D y Fondos Estructurales. (Vallmitjana i Palau, 2008).

Según Curto (2012), en el escenario mundial, los emprendedores sociales "han proliferado con fuerza" (p. 7).

Allí donde la mayoría ve un problema, ellos ven una oportunidad para contribuir a la mejora de la sociedad. De hecho, en las universidades y en las escuelas de dirección de empresas, el creciente interés por el tema se ha traducido en una mayor oferta de cursos en esta materia. [...] De este modo, existe un grupo cada vez más numeroso de emprendedores altamente formados para hacer frente, de manera innovadora y eficiente, a las carestías de la sociedad, a la vez que también son mayores los recursos disponibles para este fin.

Por su parte, algunos gobiernos han visto en estos agentes una alternativa para paliar su falta de recursos y eficiencia para financiar el incremento en la demanda de gasto social.

En América Latina, los gobiernos de turno también han establecido diversas políticas de fomento al emprendimiento con el firme propósito de disminuir los altos niveles de inequidad social en sus poblaciones. Tales políticas, obviamente, han estado orientadas por las disposiciones establecidas por organismos y agencias multilaterales, como el Banco Interamericano de Desarrollo (BID), el Sistema Económico Latinoamericano y del Caribe (SELA) y la Organización de las Naciones Unidas para la Educación, la Ciencia y la Cultura (UNESCO), que instan a los gobiernos de este hemisferio a generar espacios para el desarrollo de todas las formas de emprendimiento.

En este sentido, en palabras de Curto (2012), "el emprendimiento implica convertir una idea nueva en una innovación exitosa utilizando habilidades, visión, creatividad, persistencia [y,] a la vez[,] exposición al riesgo" (p. 10). Además, en su texto resalta "la importancia que tiene la aplicación de métodos empresariales a iniciativas sociales. [...] [La] clave de la empresa social es 'adoptar un enfoque de tipo empresarial e innovador a la provisión de servicios comunitarios"'. (Pomerantz, 2003, p. 26, citado en Curto, 2012, p. 10). 
La referencia del citado autor es determinante a la hora de considerar el emprendimiento social como alternativa de soluciones innovadoras a los problemas sociales, soluciones que permitan promover el desarrollo tanto económico como social de una región.

Todo emprendimiento social incluye tres elementos claves: un objetivo social, una innovación transformadora y un modelo de negocios sostenible. El emprendimiento social suele darse y tener éxito en los sectores de la economía donde el mercado ha fallado y la acción del Estado es inexistente o ineficaz. (Romero, 2010).

Es de resaltar cómo el enfoque del emprendimiento social permite abordar la pluralidad y la diversidad de los esquemas económicos construidos desde lo local, ya que enfatiza en las formas comunitarias creativas que generan innovación social. El énfasis en la creatividad del emprendedor social y el ambiente en el cual están inmersos los emprendedores hacen posible tener una plataforma de análisis análoga entre el emprendimiento social y el desarrollo local, en la se rescatan dos elementos comunes desde ambos enfoques: el sujeto y el territorio.

Para Varela (2008), "los Estados y sus sociedades han llegado al convencimiento de que deben estimular cada día más la iniciativa privada" por medio de programas de apoyo "a todos aquellos que tengan capacidad de defenderse por sí mismos". Los emprendedores, entonces, deben ser incentivados y apoyados por la vía tanto de medios educacionales como de reformas administrativas.

Se pueden reconocer dos modelos principales de emprendimiento: el primero es el tradicional, relacionado con la generación de actividades que transforman o comercializan bienes o servicios bajo un interés netamente económico por parte de los inversionistas. El segundo tipo se fundamenta en el desarrollo de actividades económicas orientadas a la generación de valor en la construcción de capital social. De este último modelo se desprende el concepto de "emprendimiento social".

Kliksberg (2012) sugiere que hay una demanda creciente de políticas públicas con altos niveles de calidad y centradas en las reales prioridades de la gente. También se requieren propuestas de otros actores sociales, como los emprendedores sociales, que se caracterizan por abordar problemas sociales agudos desde una perspectiva no convencional y por crear soluciones no tradicionales para enfrentarlos.

Igualmente, se pueden resaltar las políticas públicas que benefician a los esquemas gerenciales con cierto sentido social mediante la reducción de gravámenes para su creación, la exaltación de casos exitosos y la replicación de aquellas experiencias que alcanzan un escenario superior a su propia responsabilidad social. Estas iniciativas, sean individuales o colectivas, no buscan ningún lucro, pero transcienden en el tiempo.

Hecha la consideración anterior, se debe iniciar con la formación en emprendimiento $y$ con el entrenamiento en habilidades gerenciales y gestión empresarial, orientados a los objetivos sociales, además de los económicos. Pero estos aspectos muchas veces no son suficientes para estimular cambios sociales significativos, ya sea por la ausencia del Estado o por la falta de continuidad en los programas de gobierno municipales, departamentales y/o nacionales que estimulen las iniciativas y el fortalecimiento de emprendimientos.

Sobre la base de lo planteado, es posible afirmar que en el Distrito Especial, Turístico, Histórico y Cultural de Santa Cruz de Mompox no existe un adecuado emprendimiento social ni en el área central ni en las zonas 
de frontera, pues no se cuenta con incentivos para desarrollar emprendimientos, lo que lleva, a su vez, a un escaso desarrollo económico. Otro factor importante que repercute directamente en el bajo desarrollo económico de este municipio se asocia con los débiles esquemas de asociatividad empresarial de sus habitantes. Además, se evidencian escasos conocimientos para ofrecer servicios excelentes a los turistas y que los motiven a volver. Así, pues, en muchos casos se carece de una oferta de productos y servicios turísticos lo suficientemente atractiva para los clientes. En síntesis, el escaso fomento empresarial y la carencia de competencias emprendedoras e innovadoras impiden fortalecer las unidades de negocios turísticos en la zona.

En virtud de lo anterior, pueden crearse oportunidades de mejora a los emprendimientos sociales en Santa Cruz de Mompox y pueden impulsarse otros nuevos, los cuales deben estar orientados a sus características, como la comunicación, la innovación, la oportunidad, las relaciones interpersonales, esenciales para generar valor social; de igual manera, han de diseñarse y ejecutarse estrategias empresariales que permitan la vigencia de tales emprendimientos.

En Mompox, el sector empresarial del turismo es considerado como eje impulsor del crecimiento económico del municipio y sus alrededores no solo por su capacidad de generar empleos, sino también por conformar un eslabón fundamental en la cadena de producción, teniendo en cuenta el siguiente aspecto:

El incremento sostenido de la competitividad se constituye, en la actualidad, en un requisito indispensable para el crecimiento y para la propia viabilidad [...] del sector. En contraste, la sencillez del modelo organizativo de las [empresas] les puede facilitar el desarrollo de una ventaja competitiva, que le[s] permite construir eficientemente el tipo de flexibilidad más adecuada para sus estrategias competitivas; "en la medida en que las organizaciones se hacen flexibles, la competencia las va obligando a ser ágiles, la flexibilidad entonces se convierte en el requisito para competir para responder más rápido que los competidores". (Rincón y Romero, 2002; Solano, 2017).

Desde esta perspectiva, directivos de estas instituciones deben concebir estas organizaciones [turísticas] como [...] ente[s] en evolución permanente caracterizadas por la flexibilidad en sus procesos, agilidad en sus funciones, [y] la adopción de estrategias ajustadas a las cambiantes necesidades del entorno.

Asimismo, se requiere incorporar una cultura organizacional sustentada en valores que provenga del consenso de los miembros de la organización, para que estos la internalicen y se logre la transformación de la gestión empresarial. Una estrategia adecuadamente formulada ayuda a poner orden y [a] asignar, con base tanto en sus atributos como en sus deficiencias internas, los recursos de una organización, con el fin de lograr una situación viable y original, así como anticipar los posibles cambios en el entorno. (Solano, 2017, p. 10).

Continuando con lo planteado sobre el emprendimiento social, para promover el turismo cultural y ecológico en el Distrito Especial, Turístico, Histórico y Cultural de Santa Cruz de Mompox han de implementarse una serie de estrategias que le permitan ganar, empezando por una excelente gestión directiva, que posibilite buenas tomas de decisiones en las empresas dedicadas al sector turismo. Así mismo, han de tenerse valores compartidos, encaminados a un liderazgo organizacional. 
Santa Cruz de Mompox cuenta con un gran potencial turístico, y en la última década se ha constituido en un municipio turístico sostenible; pero, a pesar de ello, existen las debilidades propias de estos exóticos lugares rurales, las cuales han generado una baja tendencia a ser visitados. Esto implica que se deben buscar estrategias que fortalezcan el turismo en la región, ya que el desarrollo económico de las comunidades que allí habitan puede valorizarse con el incremento del comercio, de hoteles y restaurantes, $\mathrm{y}$, por consiguiente, potenciar la importancia de la región como atractivo turístico ante el turista.

En la presente investigación se sugiere promover el emprendimiento de actividades dirigidas al desarrollo del turismo rural comunitario en el Distrito objeto de estudio. Este tipo de turismo cuenta con el respaldo de las políticas públicas propuestas por el Estado para el desarrollo sostenible; si se potencia este tipo de actividad productiva en Mompox, se podrían mejorar las condiciones de vida de la comunidad que habita en el territorio, todo con miras a buscar la formación de una sociedad sustentable. Por esta razón, se propone el fortalecimiento de la innovación en las empresas del sector turismo del Distrito Especial, Turístico y Cultural de Santa Cruz de Mompox.

\section{Metodología}

Villa (2016) desarrolló la investigación Diseño de plan estratégico para promocionar el turismo en la provincia de Chimborazo para optar al título de magíster en Administración de Empresas por la Universidad de Guayaquil.

El método utilizado fue de observación[,] permite observar las causas y efectos del problema y llegar a una conclusión, las técnicas empleadas dirigidas a prestadores de servicios y turistas, evidenciándose la importancia de la planificación estratégica de turismo a través de (sic) las comunidades, mediante un proceso sistemático para la evolución del turismo comunitario y un futuro comprometido con los participantes de la empresa comunitaria y con la demanda al brindar un servicio de calidad. (Villa, 2016, p. 9).

Los resultados de la investigación, obtenidos por medio del análisis DOFA y de las visitas de observación, hicieron posible desarrollar estrategias competitivas que permitieran "contribuir con las entidades públicas, tales como [los] gobierno[s] locales (municipios), mediante la sistematización de programas turísticos ofertando servicios de alta calidad a los turistas" (Villa, 2016, pp. 42-43). Por lo tanto, el diseño del plan estratégico propuesto tenía como objetivo

consolidar el turismo comunitario como eje articulador del desarrollo de la provincia de Chimborazo, que contribuya a la economía de los habitantes generando fuentes de trabajo [y] permitiendo tener una vida digna, a generar las condiciones necesarias para mejorar la competitividad en la región. (Villa, 2016, p. 66).

La investigación de Villa (2016) guarda una relación directa con el presente estudio, más si se tiene en cuenta que ambas persiguen el mismo objetivo: promocionar el turismo en una zona cultural e histórica. Por tal razón, la investigación de Villa aporta a la fundamentación teórica y a la metodología que orientan conceptualmente este trabajo.

De otra parte, Rosas (2016) realizó la investigación Planeamiento estratégico y su repercusión en la gestión empresarial del sector turismo de la región Puno, 2015-2016 para optar al título de magíster en Administración de Empresas de la Universidad Andina Néstor Cáceres Velásquez, en Perú. En lo referente a la metodología que Rosas siguió en su investigación, la autora explica: 
El método para nuestro trabajo de investigación que se utilizó es el análisis y síntesis. (p. 109).

$[\ldots]$

La presente investigación es de nivel explicativo causal porque su finalidad es determinar el grado de relación o asociación (causal) existente entre dos o más variables. En estos estudios van (sic) más allá de la descripción de conceptos o fenómenos o de las relaciones entre conceptos; es decir, están dirigidas a responder por las causas de los eventos y fenómenos físicos o sociales.

El diseño de la investigación es no experimental, transaccional o transversal. No existe manipulación activa de alguna variable y se busca establecer la relación de variables medidas en una muestra en un único momento del tiempo.

La población se tomó de las empresas que brindan servicio en el sector turismo[,] entre ellos (sic) tenemos hoteles (55), hostales categorizados (61), hostales no categorizados (235), restaurantes (144) y agencias de viajes (85).

En virtud de lo anterior se tomó muestra probabilística de 100 empresas del sector. Para la recolección de la información se diseñó un instrumento tipo encuesta, el cual se dividió así[:] para [...] obtener información mediante el instrumento de planteamiento estratégico tuvo 40 ítems y en su dimensión [de] participación constó de 13 preguntas[,] y en la evaluación de 8. Continuando con la segunda variable[,] gestión empresarial tuvo de (sic) una composición de 20 preguntas.

Con respecto a los resultados de la investigación de Rosas, se evidenció correlación entre las variables del planteamiento estratégico y gestión empresarial (Rosas, p. 119), aunque se comprobó que las empresas en la región de estudio presentaron un nivel medio en el planteamiento estratégico; por otro lado, con respecto a la gestión empresarial, esta resultó ser buena.

De otra parte, César Sarasara realizó en 2015 la investigación titulada El planeamiento estratégico del turismo ecológico en América Latina: caso Posada Amazonas del Perú y del Ecuador para obtener el grado académico de doctor en Ciencias Administrativas. Su objetivo fue determinar los ejes del ecoturismo que forman parte del planeamiento estratégico del ecoturismo en América Latina, más específicamente, en Posada Amazonas.

La metodología utilizada en su estudio "fue cualitativa y cuantitativa, de lógica deductiva y correlacional. Así mismo, la investigación fue no experimental y transversal" (Sarasara, 2015, p. 137). "El análisis de los datos se realizó mediante el método multivariado, haciendo uso del análisis factorial[;] con el método se ha[n] seleccionado las variables de mayor puntuación en cada grupo; también se usó (sic) las técnicas del planeamiento estratégico" (p. xviii).

De igual manera, el análisis realizado por Sarasara (2015) permitió determinar lo siguiente:

[L]as comunidades nativas no están conformes con la instalación de empresas hidrocarburíferas, que dañan el ecosistema, mientras [que sí] apoyan el crecimiento y [la] creación de otras empresas de turismo ecológico. Se concluye que el planeamiento estratégico para el ecoturismo en América Latina es mediante la aplicación de los ejes del ecoturismo en alianzas estratégicas. (p. xviii).

La presente investigación toma aspectos de la metodología propuesta por Villa (2016), Rosas (2016) y Solano (2017) en 
sus respectivos trabajos. El instrumento empleado para la recolección de datos consiste en un cuestionario, el cual fue aplicado a treinta empresas del sector turismo ubicadas en el Distrito Especial, Turístico, Histórico y Cultural de Santa Cruz de Mompox. En la elaboración del cuestionario se tuvo en cuenta la variable "planificación estratégica" y se tomaron en consideración dos dimensiones: "fases de la planificación estratégica" y "tipos de planificación estratégica". En cuanto a los indicadores considerados, para la primera dimensión estos fueron "misión", "visión" y "objetivos" empresariales; para la segunda, "planificación corporativa", "planificación técnica o funcional" y "planificación operativa".

\section{Resultados}

Con la investigación se pretende incentivar y motivar a Mompox para que utilice un inventario que ayude a referenciar otros estudios que orienten la consecución del desarrollo integral del municipio, impulse los emprendimientos culturales y ecológicos y a la vez preserve las tradiciones históricas en pro de generar beneficio a la comunidad local.

En el ámbito social, la presente investigación es de gran interés, puesto que se preocupa por esta variable, a partir de la cual se harán replanteamientos sobre el rol que deben asumir los emprendimientos sociales ante el entorno en el cual se encuentran inmersos. A dichos emprendimientos se les pretende dar un espaldarazo con esta investigación con el fin de que puedan lograr sus metas.

Finalmente, la importancia de diseñar un plan estratégico radica en que por medio de él se pueden emprender acciones en conjunto con el gobierno departamental y distrital para la ejecución de proyectos útiles y viables para el progreso sustentable tanto económico como social, ambiental y cultural de la zona objeto de estudio.

Se presentan a continuación los resultados del estudio, llevado a cabo con base en la propuesta metodológica de Villa (2016), Sarasara (2015), Solano (2017) y Rosas (2016). Se sigue un tratamiento estadístico descriptivo de los datos obtenidos a partir del instrumento de recolección empleado: cuestionario. La variable estudiada es "planificación estratégica". Las dimensiones sobre las cuales se indagó fueron "fases de la planificación estratégica" y "tipos de planificación estratégica"; en la primera de estas dimensiones se consideraron como indicadores la misión, la visión y los objetivos de la empresa, mientras que en la segunda los indicadores tomados en cuenta fueron la planificación corporativa, la técnica o funcional y la operativa.

Para realizar el procedimiento estadístico descriptivo, en las respuestas obtenidas por parte de la población evaluada se aplicaron operaciones estadísticas descriptivas a partir de las distribuciones de dos tipos de frecuencias: absolutas $(\mathrm{Fa})$ y relativas $(\mathrm{Fr} \%)$.

El análisis de las frecuencias absolutas y relativas de los distintos valores de la variable y de las dimensiones estudiadas dirige a una información valiosa para el presente estudio, por medio de la cual es posible diferenciar las distintas distribuciones y características del objeto de investigación. Se hace la debida comparación e interpretación de los resultados mediante la aplicación del baremo de comparación planteado con los rangos y las categorías para la variable objeto de estudio.

Con las tablas expuestas a continuación se da respuesta a los puntajes obtenidos en los diferentes análisis realizados, interpretando la variable "planificación estratégica"; también se establecen elementos estratégicos en la investigación. 
Con relación al objetivo "diagnosticar las actividades turísticas del Distrito Especial, Turístico, Histórico y Cultural de Santa Cruz de Mompox", se tiene lo siguiente:

Variable: Planificación estratégica

Dimensión: Fases de la planificación estratégica

La tabla 1 muestra las respuestas de los encuestados con respecto a la adecuada aplicación del indicador "misión" en sus empresas. Como se evidencia, la mayoría de los participantes $(64 \%)$ optaron por las respuestas "totalmente de acuerdo" y "de acuerdo", lo que implica la aplicación medianamente eficiente de este indicador en las empresas. Por su parte, el 35,4\% considera que sus empresas no cumplen de manera adecuada con este indicador. Esto indica que las empresas que participaron en el estudio deben diseñar y emplear mecanismos que promuevan la misión de la empresa como fundamento para lograr el éxito.

Tabla 1. Indicador: Misión

\begin{tabular}{|c|c|c|c|c|c|c|c|c|c|c|}
\hline \multirow{2}{*}{$\begin{array}{l}\text { Respuesta / } \\
\text { Ittems }\end{array}$} & \multicolumn{2}{|c|}{$\begin{array}{l}\text { Totalmente de } \\
\text { acuerdo (5) }\end{array}$} & \multicolumn{2}{|c|}{ De acuerdo (4) } & \multicolumn{2}{|c|}{$\begin{array}{l}\text { Ni de acuerdo ni } \\
\text { en desacuerdo (3) }\end{array}$} & \multicolumn{2}{|c|}{ En desacuerdo (2) } & \multicolumn{2}{|c|}{$\begin{array}{c}\text { Totalmente en } \\
\text { desacuerdo (1) }\end{array}$} \\
\hline & $\mathrm{Fa}$ & $\mathrm{Fr}$ & $\mathrm{Fa}$ & $\mathrm{Fr}$ & $\mathrm{Fa}$ & $F r$ & $\mathrm{Fa}$ & $\mathrm{Fr}$ & $\mathrm{Fa}$ & $F r$ \\
\hline 1 & 20,0 & 41,7 & 16,0 & 33,3 & 0,0 & 0,0 & 12,0 & 25,0 & 0,0 & 0,0 \\
\hline 2 & 19,0 & 39,6 & 17,0 & 35,4 & 2,0 & 4,2 & 10,0 & 20,8 & 0,0 & 0,0 \\
\hline 3 & 0,0 & 0,0 & 21,0 & 43,8 & 24,0 & 50,0 & 1,0 & 2,1 & 2,0 & 4,2 \\
\hline Promedio & 13,0 & 27,1 & 18,0 & 37,5 & 8,7 & 18,1 & 7,7 & 16,0 & 0,7 & 1,4 \\
\hline Media & \multicolumn{10}{|c|}{3,78} \\
\hline Desv. Est. & \multicolumn{10}{|c|}{1,17} \\
\hline
\end{tabular}

Fuente. Elaboración propia (2018).

Tabla 2. Indicador: Visión

\begin{tabular}{|c|c|c|c|c|c|c|c|c|c|c|}
\hline \multirow{2}{*}{ Respuesta / Ítems } & \multicolumn{2}{|c|}{$\begin{array}{l}\text { Totalmente de } \\
\text { acuerdo (5) }\end{array}$} & \multicolumn{2}{|c|}{ De acuerdo (4) } & \multicolumn{2}{|c|}{$\begin{array}{l}\text { Ni de acuerdo ni } \\
\text { en desacuerdo (3) }\end{array}$} & \multicolumn{2}{|c|}{ En desacuerdo (2) } & \multicolumn{2}{|c|}{$\begin{array}{l}\text { Totalmente en } \\
\text { desacuerdo (1) }\end{array}$} \\
\hline & $F a$ & $\mathrm{Fr}$ & $\mathrm{Fa}$ & $F r$ & $\mathrm{Fa}$ & $F r$ & $\mathrm{Fa}$ & $F r$ & $\mathrm{Fa}$ & $\mathrm{Fr}$ \\
\hline 4 & 0 & 0 & 22,0 & 45,8 & 25,0 & 52,1 & 1,0 & 2,1 & 0,0 & 0,0 \\
\hline 5 & 0,0 & 0,0 & 20,0 & 41,7 & 25,0 & 52,1 & 2,0 & 4,2 & 1,0 & 2,1 \\
\hline 6 & 2,0 & 4,2 & 19,0 & 39,6 & 24,0 & 50,0 & 1,0 & 2,1 & 2,0 & 4,2 \\
\hline Promedio & 0,7 & 1,4 & 20,3 & 42,4 & 24,7 & 51,4 & 1,3 & 2,8 & 1,0 & 2,1 \\
\hline Media & \multicolumn{10}{|c|}{3,57} \\
\hline Desv. Est. & \multicolumn{10}{|c|}{1,12} \\
\hline
\end{tabular}

Fuente: Elaboración propia (2018). 
Dado que la media ubica el indicador en un rango de 3,78 (moderadamente eficiente) y que la desviación estándar es 1,17 (dato que aleja dispersamente el indicador), se estima que las empresas deben propender a considerar este aspecto como punto de partida de su direccionamiento estratégico.

En efecto, la misión representa la identidad de la empresa, es decir, expresa su razón de ser, su naturaleza y cómo la organización se a conocer ante sus miembros. Lo que se busca con la definición de la misión es que la empresa sea percibida por todos de forma clara, precisa, sólida, sobre estándares razonables y medibles en su aplicación. Así mismo, la misión expone las actividades que la organización desarrolla, también su amplitud, sus limitaciones, responsabilidades y objetivos, entre otros aspectos, por eso todos los niveles de la empresa deben reconocerla.

Variable: Planificación estratégica

Dimensión: Fases de la planificación estratégica

La tabla 2 presenta los resultados obtenidos con respecto a la adecuada aplicación del indicador "visión" en las organizaciones. Al respecto, el 56,3\% de los encuestados optaron por las siguientes opciones de respuesta: "Ni de acuerdo ni en desacuerdo", "en desacuerdo" y "totalmente en desacuerdo". Esto indica que las empresas deben fortalecer este aspecto, tan fundamental en las organizaciones. Por su parte, el 43,7\% restante escogió las otras opciones de respuesta, lo que implica la aplicación medianamente adecuada de la visión como fundamento de la empresa. $\mathrm{La}$ media se categorizó como moderadamente eficiente, con un valor de 3,57.

La visión refleja aquello que la empresa quiere ser en el futuro. La visión corporativa ayuda a identificar con claridad los acontecimientos que la empresa desea que ocurran en el futuro y a programar dichos sucesos en un proceso de cambio dirigido hacia la mejora continua. La visión siempre se expresa en términos de éxito ante los ojos de los clientes, tanto de los internos (empleados, dueños y proveedores) como de los externos (consumidores de sus productos y/o servicios).

Variable: Planificación estratégica

Dimensión: Fases de la planificación estratégica

Tabla 3. Indicador: Objetivos

\begin{tabular}{|c|c|c|c|c|c|c|c|c|c|c|}
\hline \multirow{2}{*}{$\begin{array}{l}\text { Respuesta / } \\
\text { Items }\end{array}$} & \multicolumn{2}{|c|}{$\begin{array}{l}\text { Totalmente de } \\
\text { acuerdo (5) }\end{array}$} & \multicolumn{2}{|c|}{ De acuerdo (4) } & \multicolumn{2}{|c|}{$\begin{array}{l}\text { Ni de acuerdo ni en } \\
\text { desacuerdo (3) }\end{array}$} & \multicolumn{2}{|c|}{$\begin{array}{c}\text { En desacuerdo } \\
\text { (2) }\end{array}$} & \multicolumn{2}{|c|}{$\begin{array}{l}\text { Totalmente en } \\
\text { desacuerdo (1) }\end{array}$} \\
\hline & $\mathrm{Fa}$ & $\mathrm{Fr}$ & $\mathrm{Fa}$ & $\mathrm{Fr}$ & $\mathrm{Fa}$ & $\mathrm{Fr}$ & $\mathrm{Fa}$ & $\mathrm{Fr}$ & $\mathrm{Fa}$ & $\mathrm{Fr}$ \\
\hline 7 & 15 & 31,25 & 18,0 & 37,5 & 10,0 & 20,8 & 5,0 & 10,4 & 0,0 & 0,0 \\
\hline 8 & 2,0 & 4,2 & 18,0 & 37,5 & 26,0 & 54,2 & 1,0 & 2,1 & 1,0 & 2,1 \\
\hline 9 & 2,0 & 4,2 & 16,0 & 33,3 & 24,0 & 50,0 & 5,0 & 10,4 & 1,0 & 2,1 \\
\hline Promedio & 6,3 & 13,2 & 17,3 & 36,1 & 20,0 & 41,7 & 3,7 & 7,6 & 0,7 & 1,4 \\
\hline Media & \multicolumn{10}{|c|}{3,66} \\
\hline Desv. Est. & \multicolumn{10}{|c|}{0,9} \\
\hline
\end{tabular}

Fuente: Elaboración propia (2018) 
La tabla 3 presenta los resultados obtenidos con respecto al indicador "objetivos". En este caso, se indagó acerca de si en la empresa se cumplen los objetivos propuestos. El $57 \%$ de los encuestados escogieron las opciones de respuesta "ni de acuerdo ni en desacuerdo", "en desacuerdo" y "totalmente de acuerdo", lo cual devela que los objetivos no se cumplen y que, por ende, este indicador no cumple con sus funciones en la organización. Por tal razón, las empresas que participaron en el estudio deben fortalecer este indicador, para que se supere ese 49,3\% de aceptación.

Por otro lado, la media se ubica en el baremo como moderadamente eficiente, con 3,66; de igual manera, tiene un dispersión de 0,99 , lo cual es significativo. Las organizaciones deben propiciar espacios que les permitan cumplir con sus objetivos estratégicos.

$\mathrm{Al}$ analizar la dimensión "fases de la planificación estratégica", se observa que la media se ubica en 3,67 y que en el baremo se categoriza como "medianamente eficiente". Esto indica que las empresas deben propiciar espacios para el desarrollo de esta dimensión como factor preponderante en este nuevo mileno.

\section{Variable: Planificación estratégica}

Dimensión: Tipos de planificación estratégica

Tabla 4. Análisis de la dimensión "fases de la planificación estratégica"

\begin{tabular}{|c|c|c|c|c|c|c|c|c|c|c|c|c|}
\hline \multirow[t]{2}{*}{ Indicadores } & \multicolumn{2}{|c|}{$\begin{array}{c}\text { Totalmente de } \\
\text { acuerdo (5) }\end{array}$} & \multicolumn{2}{|c|}{ De acuerdo (4) } & \multicolumn{2}{|c|}{$\begin{array}{c}\text { Ni de acuerdo ni } \\
\text { en desacuerdo } \\
\text { (3) }\end{array}$} & \multicolumn{2}{|c|}{$\begin{array}{c}\text { En } \\
\text { desacuerdo } \\
\text { (2) }\end{array}$} & \multicolumn{2}{|c|}{$\begin{array}{l}\text { Totalmente en } \\
\text { desacuerdo (1) }\end{array}$} & \multirow[t]{2}{*}{ Media } & \multirow[t]{2}{*}{$\begin{array}{c}\text { Desv. } \\
\text { Est. }\end{array}$} \\
\hline & $\mathrm{Fa}$ & $F r$ & $F a$ & $F r$ & $F a$ & $F r$ & $F a$ & $\mathrm{Fr}$ & $F a$ & $\mathrm{Fr}$ & & \\
\hline Misión & 13,0 & 27,1 & 18 & 37,5 & 8,7 & 18,1 & 7,7 & 16,0 & 0,7 & 1,4 & 3,8 & 1,18 \\
\hline Visión & 0,67 & 1,4 & 20,3 & 42,4 & 24,7 & 51,4 & 1,3 & 2,8 & 1,0 & 2,1 & 3,6 & 1,13 \\
\hline Objetivos & 6,33 & 13,2 & 17,3 & 36,1 & 20,0 & 41,7 & 3,7 & 7,6 & 0,7 & 1,4 & 3,7 & 1,00 \\
\hline Promedio & 6,67 & 13,9 & 18,6 & 38,7 & 17,8 & 37,0 & 4,2 & 8,8 & 0,8 & 1,6 & 3,6 & 1,10 \\
\hline
\end{tabular}

Fuente: Elaboración propia (2018).

Tabla 5. Indicador: Planificación corporativa

\begin{tabular}{|c|c|c|c|c|c|c|c|c|c|c|}
\hline \multirow{2}{*}{$\begin{array}{l}\text { Respuesta/ } \\
\text { Items }\end{array}$} & \multicolumn{2}{|c|}{$\begin{array}{l}\text { Totalmente de } \\
\text { acuerdo (5) }\end{array}$} & \multicolumn{2}{|c|}{ De acuerdo (4) } & \multicolumn{2}{|c|}{$\begin{array}{l}\text { Ni de acuerdo ni } \\
\text { en desacuerdo (3) }\end{array}$} & \multicolumn{2}{|c|}{ En desacuerdo (2) } & \multicolumn{2}{|c|}{$\begin{array}{l}\text { Totalmente en } \\
\text { desacuerdo (1) }\end{array}$} \\
\hline & $\mathrm{Fa}$ & $\mathrm{Fr}$ & $\mathrm{Fa}$ & $\mathrm{Fr}$ & $\mathrm{Fa}$ & $\mathrm{Fr}$ & $\mathrm{Fa}$ & $\mathrm{Fr}$ & $\mathrm{Fa}$ & $\mathrm{Fr}$ \\
\hline 10 & 17 & 35,4 & 16,0 & 33,3 & 3,0 & 6,3 & 10,0 & 20,8 & 2,0 & 4,2 \\
\hline 11 & 0,0 & 0,0 & 0,0 & 0,0 & 1,0 & 2,1 & 31,0 & 64,6 & 16,0 & 33,3 \\
\hline 12 & 0,0 & 0,0 & 2,0 & 4,2 & 4,0 & 8,3 & 24,0 & 50,0 & 18,0 & 37,5 \\
\hline Promedio & 5,7 & 11,8 & 6,0 & 12,5 & 2,7 & 5,6 & 21,7 & 45,1 & 12,0 & 25,0 \\
\hline Media & \multicolumn{10}{|c|}{2,68} \\
\hline Desv. & \multicolumn{10}{|c|}{1,05} \\
\hline
\end{tabular}

Fuente: Elaboración propia (2018). 
En lo referente al indicador "planificación corporativa", se indagó entre los encuestados acerca de si los procesos para la planificación estratégica corporativa son suficientes en sus empresas. Los datos revelan que las opciones de respuesta escogidas fueron "ni de acuerdo ni en desacuerdo", "en desacuerdo" y "totalmente en desacuerdo". Ello refleja que para el $75,7 \%$ dichos procesos no resultan eficientes, de manera que las empresas están llamadas a formular estrategias que propendan a mejorar este aspecto. Se destaca que para el $24,3 \%$ de los participantes en el estudio este indicador sí se cumple en sus organizaciones.

Con respecto a la media, esta se ubica en el baremo con 2,66, lo cual indica que se debe mejorar considerablemente este aspecto en las organizaciones. La media tiene una dispersión moderada de 1,05 , lo cual sugiere que las empresas del sector turismo de la zona deben propender a mejorar sus acciones estratégicas.

\section{Variable: Planificación estratégica}

Dimensión: Tipos de planificación estratégica

Los resultados obtenidos en referencia al indicador "planeación táctica" revelan que para el 75,6\% de los encuestados los procesos de la planificación táctica para el manejo gerencial son poco eficientes en sus empresas, pues optaron por las opciones de respuesta "ni de acuerdo ni en desacuerdo", "en desacuerdo" y "totalmente en desacuerdo". De manera que las empresas están llamadas a formular estrategias que propendan a mejorar este aspecto, aunque se destaca que para el $24,3 \%$ tales procesos sí resultan eficientes, de modo que se cumple con el indicador. Con respecto a la media, esta se ubica en el baremo con 2,66, lo cual señala que se ha de mejorar considerablemente en este aspecto; la media tiene una dispersión moderada de 1,05. La planificación táctica juega un papel fundamental para mejorar de manera sustancial las decisiones gerenciales que se toman desde los cargos directivos.

Variable: Planificación estratégica

Dimensión: Tipos de planificación estratégica

En relación con la tabla 7, se indagó la existencia de planificación operativa en las empresas. Al respecto, la población encuestada seleccionó las opciones de respuesta "ni de acuerdo ni en desacuerdo", "en desacuerdo" y "totalmente en desacuerdo", con un porcentaje de 79,2\%. Los resultados indican que el nivel de planificación operativa en las empresas es bajo, de modo que estas deben

Tabla 6. Indicador: Planificación táctica o funcional

\begin{tabular}{|c|c|c|c|c|c|c|c|c|c|c|}
\hline \multirow{2}{*}{$\begin{array}{l}\text { Respuesta / } \\
\text { Items }\end{array}$} & \multicolumn{2}{|c|}{$\begin{array}{l}\text { Totalmente de } \\
\text { acuerdo (5) }\end{array}$} & \multicolumn{2}{|c|}{ De acuerdo (4) } & \multicolumn{2}{|c|}{$\begin{array}{l}\text { Ni de acuerdo ni } \\
\text { en desacuerdo (3) }\end{array}$} & \multicolumn{2}{|c|}{ En desacuerdo (2) } & \multicolumn{2}{|c|}{$\begin{array}{l}\text { Totalmente en } \\
\text { desacuerdo (1) }\end{array}$} \\
\hline & $\mathrm{Fa}$ & $F r$ & $\mathrm{Fa}$ & $F r$ & $\mathrm{Fa}$ & $F r$ & $\mathrm{Fa}$ & $F r$ & $\mathrm{Fa}$ & $F r$ \\
\hline 13 & 0 & 0 & 4,0 & 8,3 & 3,0 & 6,3 & 23,0 & 47,9 & 18,0 & 37,5 \\
\hline 14 & 0,0 & 0,0 & 24,0 & 50,0 & 19,0 & 39,6 & 5,0 & 10,4 & 0,0 & 0,0 \\
\hline 15 & 0,0 & 0,0 & 2,0 & 4,2 & 5,0 & 10,4 & 23,0 & 47,9 & 18,0 & 37,5 \\
\hline Promedio & 0,0 & 0,0 & 10,0 & 20,8 & 9,0 & 18,8 & 17,0 & 35,4 & 12,0 & 25,0 \\
\hline Media & \multicolumn{10}{|c|}{2,66} \\
\hline Desv. & \multicolumn{10}{|c|}{1,13} \\
\hline
\end{tabular}

Fuente: Elaboración propia (2018). 
Tabla 7. Indicador: Planificación operativa

\begin{tabular}{|c|c|c|c|c|c|c|c|c|c|c|}
\hline \multirow{2}{*}{$\begin{array}{l}\text { Respuesta / } \\
\text { Items }\end{array}$} & \multicolumn{2}{|c|}{$\begin{array}{l}\text { Totalmente de } \\
\text { acuerdo (5) }\end{array}$} & \multicolumn{2}{|c|}{ De acuerdo (4) } & \multicolumn{2}{|c|}{$\begin{array}{l}\text { Ni de acuerdo ni } \\
\text { en desacuerdo (3) }\end{array}$} & \multicolumn{2}{|c|}{ En desacuerdo (2) } & \multicolumn{2}{|c|}{$\begin{array}{l}\text { Totalmente en } \\
\text { desacuerdo (1) }\end{array}$} \\
\hline & $\mathrm{Fa}$ & $\mathrm{Fr}$ & $\mathrm{Fa}$ & $\mathrm{Fr}$ & $\mathrm{Fa}$ & $F r$ & $\mathrm{Fa}$ & $\mathrm{Fr}$ & $\mathrm{Fa}$ & $F r$ \\
\hline 16 & 0,0 & 0,0 & 0,0 & 0,0 & 23,0 & 47,0 & 24,0 & 50,0 & 1,0 & 2,1 \\
\hline 17 & 0,0 & 0,0 & 1,0 & 2,1 & 3,0 & 6,3 & 24,0 & 50,0 & 20,0 & 41,0 \\
\hline 18 & 0,0 & 0,0 & 1,0 & 2,1 & 19,0 & 39,0 & 24,0 & 50,0 & 4,0 & 8,3 \\
\hline Promedio & 0,0 & 0,0 & 0,7 & 1,4 & 15,0 & 31,0 & 24,0 & 50,0 & 8,3 & 17,0 \\
\hline Media & \multicolumn{10}{|c|}{2,54} \\
\hline
\end{tabular}

Fuente: Elaboración propia (2018).

propender a mejorar este aspecto. Tan solo el 20,8\% de los encuestados escogieron las demás opciones de respuesta.

\section{Conclusiones}

Finalmente, se concluye que la planificación estratégica influye de manera directa en la gestión empresarial. Pero si en Mompox no se realizan procesos de planificación para promoción del turismo, la actividad turística no contribuirá al desarrollo de la región.

Con relación al estudio, se hace necesario impulsar el turismo de Mompox mediante un direccionamiento estratégico encaminado al turismo cultural y ecológico, que promueva su desarrollo y mejore las condiciones del entorno.

\section{Recomendaciones}

En el aspecto cultural, para el municipio de Mompox se pretende que se preserven y conserven las costumbres y tradiciones que caracterizan a los momposinos, para contribuir así a la mejora de la calidad de vida de los pobladores de Mompox mediante un desarrollo turístico viable sostenible y sustentable tanto económica como ambientalmente. Para ello, se requiere preparar al habitante del territorio momposino en el ecodesarrollo, que sea trabajado desde la planeación con un enfoque en el tiempo.

\section{Referencias}

Aguilar, S. y Ocampo, A. (coords.). (2014). De emprendedor a empresario: haga que su negocio isea negocio! México: Grupo Editorial Patria.

Aguilera, M., Bernal, C. y Quintero, P. (2006). Turismo y desarrollo en el Caribe colombiano (Serie Documentos de Trabajo sobre Economía Regional, n. $\left.{ }^{\circ} 79\right)$. Cartagena: Banco de la República. https://www.banrep.gov.co/sites/default/ files/publicaciones/archivos/DTSER-79.pdf

Alcaraz, R. (2015). El emprendedor de éxito: guía de planes de negocios (5. ${ }^{\mathrm{a}}$ ed.). México, D. F.: Editorial McGraw-Hill Interamericana.

Alemany, L., Álvarez, B., Planellas, M. y Urbano, D. (2011). Libro blanco de la iniciativa emprendedora. Barcelona: Fundación Príncipe de Gerona.

Altamira, R. y Muñoz, X. (2007). El turismo como motor de crecimiento económico. Anuario Jurídico y Económico Escurialense, XL, 677-710.

Alvarado, O. (2000). Elementos de la administración general. Lima: Editorial Udegraf S. A.

Amador, F. (10 de abril de 2002). La planeación estratégica en el proceso administrativo. 
Gestiópolis. https://www.gestiopolis.com/la-planeacion-estrategica-en-el-proceso-administrativo/

Aparicio, M. S. (2013). El reto del turismo en los espacios naturales protegidos españoles: la integración entre conservación, calidad y satisfacción [Tesis doctoral, Universidad Complutense de Madrid]. Repositorio de la UCM. https://eprints. ucm.es/20836/

Arciniegas, I. (2004). Formación profesional para el turismo colombiano. Revista Javeriana, $710,34-41$

Arrieta, C., Solís, J. A. y Zapatero, G. (2010). Planeamiento estratégico para el desarrollo turístico del valle de Lurín [Tesis de maestría, Pontificia Universidad Católica del Perú]. Repositorio de la PUCP. http://hdl.handle.net/20.500.12404/4550

Barbosa, M. (2007). La política del turismo cultural y el diseño de producto turístico para el turismo cultural. Caso: corredor turístico Bogotá-Boyacá-Santander. Revista Escuela de Administración de Negocios, 60, 105-122. https:// doi.org/10.21158/01208160.n60.2007.407

Bavaresco, A. (2010). Proceso metodológico en la investigación (4. ${ }^{\mathrm{a}}$ ed.). Maracaibo: Editorial Ediluz.

Bertoni, M. (2008). Turismo sostenible: su interpretación y alcance operativo. Cuadernos de Geografia-Revista Colombiana de Geografia, 17, 155-163. https://doi.org/10.15446/rcdg.n17.10925

Burgos, R. (2016). El turismo comunitario como iniciativa de desarrollo local. Caso localidades de Ciudad Bolívar y Usme, zona rural de Bogotá. Hallazgos, 13(26), 193-214. http:// dx.doi.org/10.15332/s1794-3841.2016.0026.08

Burgos, R. y Cardona, M. (2014). Turismo comunitario, como estrategia para el empoderamiento comunitario en las localidades de Ciudad Bolívar, Usme y Sumapaz, zona rural. Bogotá,
D. C. Teoría y Praxis Investigativa, 9(2), 94-114. https://revia.areandina.edu.co/index.php/Pp/ article/view/418/451

Cabo, J. (2014). Gestión de la calidad en las organizaciones sanitarias. Madrid: Ediciones Díaz de Santos.

Carbajal, D. F. (2005). El planeamiento estratégico como instrumento de desarrollo del sector turismo en el Perú [Tesis de maestría, Universidad Nacional Mayor de San Marcos]. Repositorio de la Universidad Nacional Mayor de San Marcos. http://cybertesis.unmsm.edu. pe/bitstream/handle/20.500.12672/2708/carbajal_rd.pdf?sequence $=1 \&$ isAllowed $=y$

Casasola, L. (2006). Turismo y ambiente. México, D. F.: Trillas.

Ceballos-Lascuráin, H. (2003). Ecoturismo y desarrollo sostenible (1. ${ }^{\mathrm{a}}$ ed.). México: Diana S. A.

Chávez, N. (2010). Introducción a la investigación educativa (4. ${ }^{\mathrm{a}}$ reimpr.). Zulia: s/ed.

Chen, C. (2012). Design for the environment: A quality-based model for green producto development. Management Science, 47(2). https://doi. org/10.1287/mnsc.47.2.250.9841

Chiavenato, I. (2002). Administración en los nuevos tiempos. Bogotá: McGraw-Hill.

(2012). Introducción a la teoría general de la administración (8. ${ }^{\mathrm{a}}$ ed.) México: McGrawHill Interamericana.

Clark, J. (1997). A framework of approaches to sustainable tourism. Journal of Sustainable Tourism, 5(3), 224-223.

Congreso de Colombia. (12 de marzo de 2008). Ley 1185 de 2008. Por la cual se modifica y adiciona la Ley 397 de 1997 -Ley General de 
Cultura- y se dictan otras disposiciones. D. O. 46.929, del 12 de marzo de 2008. http:// www.secretariasenado.gov.co/senado/basedoc/ ley_1185_2008.html

(7 de agosto de 1997). Ley 397 de 1997. Por la cual se desarrollan los artículos 70, 71 y 72 y demás artículos concordantes de la Constitución Política y se dictan normas sobre patrimonio cultural, fomentos y estímulos a la cultura, se crea el Ministerio de la Cultura y se trasladan algunas dependencias. D. O. 43.102, del 7 de agosto de 1997. http://www.secretariasenado.gov.co/senado/ basedoc/ley_0397_1997.html

Curto, M. (2012). Los emprendedores sociales: innovación al servicio del cambio social (Cuadernos de la Cátedra "La Caixa" de Responsabilidad Social de la Empresa y Gobierno Corporativo, $n .{ }^{\circ}$ 13). Barcelona: IESE Business School y Universidad de Navarra.

De Ávila, B., Pérez, H. y Vega, R. (2016). Gestión financiera y competitividad de las empresas prestadoras de servicios turísticos en la frontera colombo-venezolana. FACE, 16(1), 38-53.

Diez, D. (2011). La planificación estratégica en espacios turísticos de interior: claves para el diseño y formulación de estrategias competitivas. Investigaciones Turísticas, 1, 69-92.

Femenía, O. (2011). La imagen de un destino turístico como herramienta de marketing. Málaga: Universidad de Málaga. https://www.eumed.net/ libros-gratis/2011c/986/index.htm

Hall, M. y Page, S. (2002). The geography of tourism and recreation: Environment, place and space. London: Routledge.

Kliksberg, B. (2012). La crisis y la responsabilidad social empresarial. En J. I. Galán Zazo y A. Sáenz de Miera (eds.), Reflexiones sobre la responsabilidad social corporativa en el siglo XXI (pp. 47-68). Salamanca: Ediciones Universidad de Salamanca.
Organización Mundial del Turismo (OMT). (1994). Planificación turística nacional y regional: metodologías y estudios de caso. Madrid: OMT.

. (2014). Panorama OMT del turismo internacional. Edición 2014. Madrid: OMTUNwTo. https://www.e-unwto.org/doi/ pdf/10.18111/9789284416202

Pomerantz, G. M. (2003). The business of social entrepreneurship in a "down economy". Business, 25(3), 25-30.

Rincón, D. y Romero, M. C. (2002). Tendencias organizacionales de las empresas. Revista Venezolana de Gerencia, 7(19), 355-374. https://www. redalyc.org/pdf/290/29001902.pdf

Romero, J. (30 de mayo de 2010). Emprendimiento social [Entrada de blog]. Siscapem Blog. https://siscapem.wordpress.com/2010/05/30/411/

Rosas, N. (2016). Planeamiento estratégico y su repercusión en la gestión empresarial del sector turismo de la región Puno, 2015-2016 [Tesis de Maestría, Universidad Andina Néstor Cáceres Velásquez]. Repositorio de la Universidad Andina Néstor Cáceres Velásquez. http://repositorio. uancv.edu.pe/handle/UANCV/628

Salcedo, A. (2013). Recuperación del turismo en Colombia [Ensayo de grado, Universidad Militar Nueva Granada]. Repositorio de la Universidad Militar Nueva Granada. https://repository.unimilitar.edu.co/bitstream/handle/10654/10873/ Ensayo\%20de\%20Grado\%20Definitivo. pdf? sequence $=1$

Sarasara, C. (2015). El planeamiento estratégico del turismo ecológico en América Latina: caso Posada Amazonas del Perú y del Ecuador [Tesis de grado, Universidad Nacional Mayor de San Marcos]. Repositorio UNMSM. https://hdl.handle. net/20.500.12672/4455

Solano, C. (2017). Responsabilidad social como elemento innovador en institución del sector 
público del departamento de La Guajira [Tesis de maestría, Universidad Privada Dr. Rafael Belloso Chacín]. Maracaibo, Venezuela. http:// virtual.urbe.edu/tesispub/0107266/

Vallmitjana i Palau, N. (2008). La actividad emprendedora de los graduados IQS [Tesis doctoral, Universirat Ramón Llull]. Barcelona, España.

Varela, R. (2008). Innovación empresarial: arte y ciencia en la creación de empresas. Bogotá: Pearson Educación.

Villa, D. M. (2016). Diseño de plan estratégico para promocionar el turismo en la provincia de
Chimborazo [Tesis de maestría, Universidad de Guayaquil]. Repositorio de la U. de Guayaquil. http://repositorio.ug.edu.ec/bitstream/ redug/17174/1/DISE $\%$ C $3 \% 910 \% 20 \mathrm{DE} \% 20$ PLAN\%20ESTRATEGICO\%20PARA\%20 PROMOCIONAR\%20EL\%20TURISMO\%20 EN\%20LA\%20PROVINCIA\%20DE\%20CHIMBORAZO.pdf

World Tourism Organization. (2011). Tourism towards 2030 / Global. Advance edition presented at UNWTO 19th General Assembly-10 October 2011, UnwTO, Madrid, https://doi. org/10.18111/9789284414024 